fungal species richness of colon polyps decreased, and the species community distribution was significantly shifted. The abundance of Ascomycete in patients was up to $50.9 \%$, of which Xylaria was three times higher than the control. In addition, pathogens such as Candida, Saccharomyces, Passalora et al are also doubled in patients. Further, combining Lefse analysis, four fungi of Candida, Xylaria, Saccharomyces and Aspergillus were significantly increased in patients, and the abundance in different individuals was relatively stable. The ROC curve was used to analyze the sensitivity and specificity of these four species to discover colon polyps, and the AUCs were obtained as $0.89583,0.62037,0.65714,0.66667$ with the accuracies of $0.857,0.8,0.667,0.7333$. In order to improve the specificity of the model, these four species were simultaneously used in the model, and the AUC was 0.75 with an accuracy of 0.667 , which up to the clinical laboratory standard. However, there were no differences in fungal community structure between patients with different pathological types and number.

Conclusions In summary, this study investigated the fungal community structure of polyps and elucidate the characteristics of patients with pathogenic fungi. What's more, this subject has established innovative intestinal microorganisms as polyps screening biomarkers.

\section{IDDF2019-ABS-0228 COMMUNITY ACQUIRED SPONTANEOUS BACTERIAL PERITONITIS IN CIRRHOTIC PATIENTS: MICROBIOLOGICAL DIAGNOSIS AND MOLECULAR CONCORDANCE WITH BACTEREMIA}

Bipanchi Mahanta*, Anup Das, Reema Nath. Assam Medical College, India

10.1136/gutjnl-2019-IDDFabstracts.202

Background About 10-30\% of Cirrhotic patients with ascites develop spontaneous bacterial peritonitis (SBP) within a year and many are community acquired. Isolation of bacteria from ascitic fluid is low to get antibiogram for choosing antibiotic. INASL recommends Ceftriaxone for empirical therapy in SBP. However, ESBL production in enteric bacteria has complicated the treatment.

Aims

1. To establish bacterial etiology of community-acquired SBP and choose a sampling procedure to enhance bacterial demonstration and isolation.

2. To determine the antibiotic susceptibility pattern of the bacterial isolates.

3. To establish the concordance between bacteria causing peritonitis and bacteremia using molecular tools.

Subjects Twenty-six consecutive clinically suspected communityacquired SBP cases, attending OPD within 6 months, in a tertiary hospital of Northeast India without the history of antibiotic therapy were enrolled.

Methods In this Cross-sectional observational study peritoneal fluid was collected in sterile container and culture bottles in duplicate with two sets of blood culture. Smears were prepared at bedside and laboratory. Culture isolates were identified conventionally. Antimicrobial sensitivity was performed using the Kirby Bauer method and tested for ESBL production. Similar Organisms from blood and ascitic fluid were confirmed by PCR using16s rRNA primers. Sanger sequencing was done and evolutionary analysis done by Maximum Likelihood method. Fisher's exact test done using GraphPad Quick Cals software.

Results Gram stains done in bedside smears showed higher $(84.6 \%)$ bacterial detection rate than done at the laboratory $(30.7 \%)$. Bacterial isolation was significantly higher $(46.42 \%)$ from Ascitic fluid collected in the culture bottle than in a sterile container (3.57\%). Escherichia coli isolation was highest (30.7\%) followed by Klebsiella pneumoniae (19.23\%) and Staphylococcus aureus (15.38\%). All Klebsiella pneumoniae and $80 \%$ of E.coli were ESBL producers and resistant to Cephalosporin and Quinolones (>80\%). Beta lactams with Beta lactamase inhibitor showed $60-70 \%$ sensitivity. Molecular and antibiogram typing showed 66\% homogeneity between similar bacteria causing SBP and bacteremia.

Conclusions Bedside smear and inoculation in suitable enrichment broth was better for bacterial detection and isolation. Bacteria causing SBP were ESBL producers warranting locally specific treatment guideline for empiric therapy. Simultaneous SBP and bacteremia by a similar organism are not rare. Further studies are needed to modify INASL guidelines.

\section{IDDF2019-ABS-0232 EFFECTS OF TERMINALIA CHEBULA ON PATIENTS WITH FUNCTIONAL DYSPEPSIA}

Sumit Rajput*, SN Sinha. Guru Gobind Singh Indraprastha University, India

\subsection{6/gutjnl-2019-IDDFabstracts.203}

Background Functional dyspepsia (FD) is a common functional gastrointestinal disorder with pain or discomfort in the upper abdomen as the main characteristic. The prevalence of FD worldwide varies between $5 \%$ and $11 \%$. This condition adversely affects attendance and productivity in the workplace. Terminalia chebula (TC) showed to have many pharmacological activities indicating anti-diabetic, cognitive enhancer, antiinflammatory, etc. This study aimed at investigating the beneficial and anti-inflammatory effect of TC on the severity and frequency of symptoms in patients with FD.

Methods In total, 220 FD patients were included in this randomized double-blind trial, based on the ROME III diagnostic criteria, and they were divided into three intervention groups namely, TC, placebo and pantoprazole. Then, the severity and frequency of symptoms during this twelve-week trial were measured. Obtained information was analyzed using Chi-square test and repeated measures test.

Results In general, the severity and frequency of symptoms after the 12th week significantly decreased in the TC group as compared to the pantoprazole and placebo groups, and continued to reduce by the end of the twelve weeks. General reduction of symptom severity and frequency in the pantoprazole group was significantly different from the placebo group by the end of the 4th and 12 th weeks. With respect to each individual symptom, TC markedly improved symptoms, such as burning, pain, early satiation, fullness, bloating, belching and nausea, as compared to the placebo-treated group. Moreover, TC significantly improved symptoms, like vomiting, and nausea, except for pain, as compared to pantoprazole treated subjects.

Conclusions According to the results, TC, as Indian traditional remedy, was more effective than pantoprazole and placebo in reducing the symptoms in FD patients. 